Running Head: IMPLEMENTATION OF HEART FAILURE CARE IN A NURSING FACILITY

\title{
Implementation of a Heart Failure Quality Initiative in a Skilled Nursing Facility: Lessons Learned Arif Nazir MD ${ }^{1}$, M. E. Dennis BS ${ }^{2}$, Kathleen T. Unroe MD, MHA ${ }^{3}$ \\ ${ }^{1}$ Indiana University School of Medicine, ${ }^{2}$ Regenstrief Institute, ${ }^{3}$ Indiana University Center for Aging Research
}

\author{
Corresponding Author: \\ Arif Nazir MD \\ Indiana University School of Medicine, \\ Indianapolis, Indiana \\ anazir@iu.edu \\ 3178806585 \\ M.E. Dennis BS \\ Regenstrief Institute \\ Indianapolis, Indiana \\ leeme@iupui.edu \\ 3172749140 \\ Kathleen T. Unroe \\ Indiana University Center for Aging Research \\ Indianapolis, Indiana \\ 317274 9235kunroe@iu.edu
}

Total Words (including references): 2470

References: 20

Abstract words: 128

Tables: 1

Figures: 1

This is the author's manuscript of the article published in final edited form as:

Nazir, A., Dennis, M. E., \& Unroe, K. T. (2015). Implementation of a heart failure quality initiative in a skilled nursing facility: lessons learned. Journal of Gerontological Nursing, 41(5), 26-33. http://doi.org/10.3928/00989134-20141216-01 


\title{
Running Head: IMPLEMENTATION OF HEART FAILURE CARE IN A NURSING FACILITY
}

\begin{abstract}
Skilled nursing facilities (SNFs) are organizations that represent complex adaptive systems, offering barriers to implementation of quality improvement (QI) initiatives. We describe our efforts, utilizing the approach of reflective adaptive process to implement a new model of care - the Skilled Heart Unit for effective heart failure (HF) care in one SNF. We convened a team of stakeholders from the local hospital system and the SNF to design and then implement this new model. Evaluation of our implementation processes confirmed the value of our strategy that centered on team-based approaches, staff engagement and flexibility of processes to respect SNF needs and culture. Interviews with facility staff revealed their perceptions that our strategy resulted in better HF care, enhanced teamwork between staff and the clinicians and improved job satisfaction. Our work provides a unique blue print of strategic QI implementation in the SNF setting.
\end{abstract}




\section{Running Head: IMPLEMENTATION OF HEART FAILURE CARE IN A NURSING FACILITY}

Lessons from the Implementation of a Heart Failure Quality Initiative in a Skilled Nursing Facility Heart Failure (HF) affects at least 20\% of patients in skilled nursing facilities (SNFs) and is a leading cause for hospitalizations (Daamen, Schols, Jaarsma, \& Hamers, 2010; Pang, Komajda, \& Gheorghiade, 2010). Evidence suggests SNFs struggle to provide adequate treatment for patients with this condition. Patients that require rehabilitation for HF in a facility after a hospital stay may be sicker than those who are discharged to the community as evident by their higher mortality rate than those who are discharged to home (Allen et al., 2011). Further, within a year of admission to the SNF, more than $40 \%$ of SNF patients with HF die and 30\% are readmitted to a hospital (Foebel et al., 2013). These poor outcomes may in part be due to underperformance in several evidence-based care components for HF care. Inconsistent implementation of evidence based guidelines, prescribing including appropriate medications, daily weights and low salt diets, has also been reported (Dolansky, Hitch, Pina, \& Boxer, 2013). Similarly, another study found that inappropriate regimens were used in more than $40 \%$ of the patients and key medications, including beta blockers and angiotensin converting enzyme inhibitors, were missing for the majority of patients with heart failure (Heckman et al., 2004). Furthermore a recent government report found that more than $30 \%$ of nursing facility residents experienced an adverse event within the first 30 days they were in the facility due to the provision of substandard care (OIG, 2014). Although best practices for HF care have been widely studied and established, approaches for implementation in SNFs are not standardized and sorely underrepresented in the literature.

SNFs represent complex adaptive systems that resist traditional approaches to quality improvement (QI) (Anderson, Issel, \& McDaniel Jr, 2003). Individuals involved in a complex adaptive system do not interact linearly; they make up a dynamic network of competition and collaboration, coevolving within their shared environment (Boustani et al., 2010). Thus, traditional top-down directives are insufficient for promoting a sustained change and a team-based, iterative QI approach is needed (Boustani, et al., 2010). Initiatives in complex environments need tailoring of processes to fit the system's unique needs and to respect the existing culture. Buy-in from the facility leadership and the frontline staff is equally important. Brainstorming solutions is facilitated by a well-functioning team of stakeholders in 


\section{Running Head: IMPLEMENTATION OF HEART FAILURE CARE IN A NURSING FACILITY}

which the roles of the team members are clearly defined (Boustani et al., 2011). This unique approach, where stakeholders meet, reflect, create and resolve conflict by dialogue in pursuit of a shared mission and vision, is called reflective adaptive process which has been shown to instill positive change in an organization (Boustani, et al., 2011; Callahan et al., 2011; Stroebel et al., 2005). A recent study highlighted the impact of stakeholder buy-in and bottom-up strategies on the successful inception and implementation of a range of QI projects in multiple SNFs in Minnesota (Arling et al., 2013).

Healthcare systems must invest in QI processes that can address barriers to effective HF care. A systematic review confirms the success of various multifaceted HF interventions, carried out by multidisciplinary teams (e.g., nurses, physicians, pharmacists, and case managers) in the inpatient and outpatient setting (McAlister, Lawson, Teo, \& Armstrong, 2001). SNFs are mandated to provide “multidisciplinary care.” However, the complexity of the patients' health problems combined with the unique stresses of the environment creates barriers to carrying out successful QI programs. Literature regarding implementation and impact of these types of initiatives is currently lacking.

In this paper, we describe experiences in designing a new model of HF care in the SNF setting utilizing principles of reflective adaptive processes to achieve common goals among stakeholders. We share lessons learned from the qualitative evaluation of the implementation of this Skilled Heart Unit.

\section{Methods}

\section{Developing the Skilled Heart Unit Model}

The initiative began with an urban, 82-bed privately owned SNF with a mix of skilled rehabilitation and long stay residents (patients who do not plan to discharge to the community) whose leadership was eager to focus on QI related to HF. The skilled rehabilitation patients have consistent nursing assignments for day shift: a registered nurse, two Licensed Practical Nurses and two Certified Nurse Aides (CNAs). Staffing varies for the evening and overnight shifts. Prior to SHU implementation, formal HF education protocols, specific HF assessments, and structured interdisciplinary discussions were not established. Moreover, use of HF clinical protocols such as daily weights was inconsistent. 


\section{Running Head: IMPLEMENTATION OF HEART FAILURE CARE IN A NURSING FACILITY}

A team of stakeholders that included the SNF medical director, nurses and administrator, along with a cardiologist, hospitalist, nurse practitioner, pharmacist and case manager at a large referring hospital was formed. Providing better HF care was the common motivation for this collaboration. For hospitals, and thus the SNFs that depend upon hospitals for their referral, reducing hospital readmissions has become an increasing priority as HF is the most common cause of hospital readmissions.

(Gheorghiade, Vaduganathan, Fonarow, \& Bonow, 2013). The group, convened and facilitated by the SNF Medical Director (AN), used the reflective adaptive process approach to meet and brainstorm for solutions to the challenges. This approach recommends that stakeholders share a common goal, address points of conflicts openly, and set aside time and space to meet frequently until the plan is finalized. (Boustani, et al., 2010) This Council met bi-monthly for a year at the hospital to develop a model of care - a SNF based Skilled Heart Unit (SHU).

While the impetus for the QI came from drive of one SNF to engage in self-improvement, the stakeholder team focused on:

-determining program protocols specific to the needs of the SNF;

•establishing delivery process and methods of monitoring the process;

•defining interactions between implementers at all levels;

•determining measures of the impact of change; and

•providing feedback from all stakeholders.

\section{Skilled Heart Unit Model of Care}

The stakeholder team reviewed literature on optimal chronic care delivery, post-discharge HF care and best practices in patient transitions to finalize the components of the model (Table 1) (McAlister, Stewart, Ferrua, \& McMurray, 2004; Naylor et al., 2004). Following the principles of the Chronic Care Model that centers on an educated patient, a proactive interdisciplinary team, and decision protocols, the SHU sought to provide care that is: (a) standardized, (b) evidence-based, and (c) proactive (Wagner et al., 2005). 


\section{Running Head: IMPLEMENTATION OF HEART FAILURE CARE IN A NURSING FACILITY}

Interdisciplinary meetings represent the most vital component of the model and have shown benefits for geriatric care in the inpatient and outpatient settings (Counsell et al., 2007; Counsell et al., 2000). Ongoing and prompt communication (face-to-face and by phone) between the staff, clinicians and hospital consultants is another key component of the SHU model, as is the systems for structured baseline and follow-up assessments of HF patients. In addition, the team at the facility created structured tools for assessments and daily care delivery to be used as documentation of care and guides for communication.

Education for staff, patients, and families is the second component of the SHU model, as it has been shown to impact health outcomes for SNF HF patients (Boxer et al., 2012). Curricula for the education of the SHU staff were adapted from the Bridge Program, an ongoing initiative in a Midwestern university hospital system, that has produced evidence of improvement in HF-related knowledge among the staff (Boxer et al., 2012). Educational resources put forth by the Heart Failure Society of America and the American Heart Association were used for patient/family education program (Lindenfeld et al., 2010). Additionally, each patient of the SHU was given a comprehensive low-salt cookbook and the opportunity to speak to the facility dietician.

Evaluation of both the program and process was emphasized with quantitative variables measuring the impact of change on the patients' health and qualitative documentation of the experiences during implementation.

As opposed to a geographic area in the SNF with dedicated beds, the SHU is a philosophy of care that aims to enhance HF care for patient located in any part of the SNF. Any patient 55 years or older, had chronic heart failure, and was able to speak and understand English was eligible for admission to SHU. While patients admitted for comfort care and/or for hospice care were excluded, patients with cognitive impairment were admitted. Over the course of the implementation and evaluation of the SHU, 15 patients consented to participate in the SHU evaluation which included medical record abstraction, collection of data regarding the use of the SHU tools, and patient and caregiver surveys of HF knowledge and satisfaction with care. Five of the patients had a level of cognitive impairment (mild, moderate, or severe) 


\section{Running Head: IMPLEMENTATION OF HEART FAILURE CARE IN A NURSING FACILITY}

and required consent from a legally authorized representative. The data collected was utilized by the implementation team for quality assurance during the course of the patients' care.

\section{Implementation of the Skilled Heart Unit}

The implementation was orchestrated by the SNF team, including the medical director, nurse practitioner, administrator, and the director of nursing, who discussed required resources and next steps. The main monetary investment of the SNF for implementation was printing educational handouts and folders for patient and family education. Added staff time for admission assessments and attendance at the interdisciplinary meetings was an additional cost of the program. These expenses were approved by the administrator who saw the SHU as an opportunity for improved quality of care and a source of positive publicity for the SNF.

Priorities for implementation were decided by the SNF-based team (medical director, registered nurse, director of nursing and facility administrator) before action was taken. Consistent with the reflective adaptive approach the team met frequently and openly discussed issues pertaining to resources needed, impact of protocols on workloads and distribution of responsibilities. The most critical strategic step was to garner prompt staff buy-in for the initiative. The SNF is reputed to provide high-quality care (rated as 5- Star rating on the Medicare Nursing Home Compare website at the time of implementation) and has dedicated and experienced staff that take pride in high performance. The facility staff was assured that implementation of the SHU would utilize the strong foundations to test a new high-quality care model. New protocols were introduced to staff as having the potential to enhance their value as team members, by assigning specific tasks and strengthening working relationships with the physician and nurse practitioner.

The registered nurse, designated as the HF Champion, oversaw day-to-day processes and also ensured that SHU tools were utilized by the nurses and nurses' aides. Figure $\mathbf{1}$ describes the care-flow and tools employed by the SHU. The HF admission assessment tool assessed baseline symptoms, function, and cognition of new patients as well as patient satisfaction with the transition to the SNF. The 


\section{Running Head: IMPLEMENTATION OF HEART FAILURE CARE IN A NURSING FACILITY}

SHU nurses used the SHU clinical rounding tool to log and compare baseline measures to daily vitals, weights and dyspnea scores. The SHU root-cause analysis tool was utilized at the interdisciplinary team meetings to assess adverse events (death and hospital transfer) and to determine opportunities for improvement.

The interdisciplinary team (medical director, nurse practitioner, dietician, therapists, SHU nurse champion and the nurses' aides) meetings occurred weekly. The team discussed the overall care plan and any changes in patients' function and cognition. Given their key role in the day to day care of residents, the inclusion of nurses’ aides was emphasized and valued during these meetings. Besides providing input in team meetings, the aides carried the "A New Leaf" pocket cards to facilitate the proactive recognition of new HF-related symptoms (Harrington, 2008).

Staff education was accomplished through biannual in-services using the Bridge Project model led by the clinicians. Education for the staff also occurred during routine interactions with the clinicians.

Patient education was provided in two sessions. The first session was conducted by the HF champion in the patient room and provided an overview of HF, medications used, and lifestyle and diet modifications. The HF champion asked the dietitian to provide more detailed instructions to the patients and their families in some cases. Handouts were used to educate and were left with the patient and their caregiver for further review. For patients with cognitive impairment, the nurse invited their caregivers to participate in the 30-minute session. The second session was provided by the nurse practitioner and/or the physician at or near the day of discharge and focused on the patient's medication regimen, warning symptoms, and the importance of lifestyle changes.

\section{Evaluation Methods}

The SHU evaluation was approved by the Indiana University Institutional Review Board, and included quantitative and a qualitative components. The quantitative data was comprised of patient and caregiver surveys collected via interviews with a research assistant along with clinical data from the nursing assessment tools. Frequent examination of this data served as quality assurance for the implementation team to monitor patient progress and patient and family satisfaction. The qualitative 


\section{Running Head: IMPLEMENTATION OF HEART FAILURE CARE IN A NURSING FACILITY}

component of the evaluation, presented here, focused on the implementation process. The research team utilized semi-structured interviews with all 5 staff members primarily involved in implementing the program to gather information about their experiences. Interviewees included the SHU champion registered nurse, two nurses’ aides, one licensed practical nurse, and the facility administrator. The interview guide was developed using examples from implementation science and included three to six questions within each of six domains (SHU procedures, HF care knowledge/abilities/skills, confidence in caring for HF, job satisfaction, patient knowledge, and overall perceptions of the program) for a total of 26 questions. The 5 interviews were conducted by the research coordinator and lasted about 30 minutes each.

\section{Results}

The verbal responses to the interview questions were audio recorded, and identifiable information was removed during transcription. The research coordinator organized the responses by each of the following themes, and trends and significant information were identified.

\section{Program Modification}

All SHU components were perceived as important, but structured communication was especially valued by staff. One respondent, based on experiences in other facilities, highlighted the need to customize the program if implemented in other SNFs. Another interviewee stressed accountability for compliance with processes including weight-taking and also to complete other processes for the HF patients, “... make sure that weights are actually getting done and that they are getting weighed the same way,” along with the advice to systematically note in patient charts when, how (standing vs. wheelchair), and who weighed the patient each day. Another suggestion was to delegate tasks for staff at the beginning of each day.

\section{Facilitators and Barriers to Implementation}

Two facilitators were unanimously reported: 1) importance of nursing education, and 2) the physician, NP, and medical director involvement with the unit processes. Four interviewees highlighted the significance of communication, particularly between the nurses' aides and the HF Champion. The 


\section{Running Head: IMPLEMENTATION OF HEART FAILURE CARE IN A NURSING FACILITY}

nurses appreciated the willingness of clinicians to ask questions of and listen to the front-line staff and believed it led to buy-in and dedication to the program.

Barriers to implementation were few and involved difficulty with patients and family members. Families were frequently noted to bring food from outside the SNF resulting patient non-compliance with diet. Reports of this barrier brought about a suggestion from staff to address it, "Non-compliant people make things difficult. They need to have the education earlier, [have] more discussions about it.”

\section{Impact on Job Satisfaction}

Interviewees observed many benefits of the project for the staff. Reports of value included improved ability to identify HF symptoms, staff empowerment, and higher job satisfaction.

\section{Efficacy of Patient and Caregiver Education}

Interviewees perceived patients and caregivers to be receptive to education and felt that handouts were valuable tools for promoting self-care. Two interviewees suggested that patient education be provided earlier and more frequently. For the non-compliant patients, one suggestion was to utilize facility dietician further to teach preparation of a heart- healthy diet.

\section{Overall Perceptions}

The importance of team work was highlighted by all respondents: "It should be everybody as a team, everybody is important.” Another said, "Everybody on the team is heard; the doctor will come to us and ask what we think. If we have questions we can go to him... Before, we [aides] would go to the nurse and the nurse would go to the doctor, but now we are all, you know, together.”

\section{Discussion}

We report on our experiences in the implementation of a QI project targeting HF care in one SNF. Aware of the difficulties in implementing QI initiatives in the complex SNF environment, we engaged a diverse group of stakeholders. Importantly, we were respectful of the resource and cultural constraints of the SNF environment. We identified an internal HF champion for the initiative and engaged the SNF staff in developing materials and protocols. The implementation evaluation affirmed the value of this collaborative approach. 


\section{Running Head: IMPLEMENTATION OF HEART FAILURE CARE IN A NURSING FACILITY}

SNFs are complex adaptive systems for which successful implementation of QI projects requires stakeholders who share goals, have dedicated meeting time and space to brainstorm strategies, and are willing to compromise (Boustani, et al., 2010). In this SHU implementation, the facility leadership and nursing staff provided active feedback into the processes and the tools resulting in grassroots support for the initiative. All relevant staff members including the frontline aides were encouraged to provide direct input to the physician and the nurse practitioner about patient care issues at daily rounds and weekly interdisciplinary meetings, thus flattening the hierarchy that usually exists in a SNF setting. The SHU implementation and its parallel evaluation offered flexibility to respond to the needs of the SNF and emphasized collaboration among disciplines. The clinicians provided leadership, clarified staff roles on the team, and facilitated communication and coordination through utilization of formal tools and processes. As found previously, the significance of team-based care, the critical role of the clinicians on the team, and communication and coordination among team members are key components for success (Nazir et al., 2013).

Staff interviews confirmed both clinician involvement and engagement of nurses’ aides as critical in program implementation. As described in the qualitative interviews, the inclusive approach to communication helped to gain the trust and confidence of the staff, generated teamwork, and improved job satisfaction. Turnover of staff is a major issue in SNFs and impacts quality of care (Horn et al., 2010), and studies show that job satisfaction impacts staff retention (Lu, While, \& Barriball, 2005). Larger investigations will be needed to confirm if such an implementation strategy truly impacts the important outcome of staff retention, however. Staff also valued patient education as an important component of the SHU model. Patient education is crucial to the chronic care model and has been shown to improve health outcomes and quality of life in patients with HF (Boyde, Turner, Thompson, \& Stewart, 2011). The interviews also provided feedback for future implementation of the SHU model. Suggestions included strategies for improving protocols and processes and stressed the importance of role clarity consistent with findings from other researchers (Lang, Thomas, Bliese, \& Adler, 2007). A larger implementation of 


\section{Running Head: IMPLEMENTATION OF HEART FAILURE CARE IN A NURSING FACILITY}

this program would likely include process accountability and task delegation along with the addition of a formal dietician component to the patient education.

Limitations to the study of the implementation include characteristics of the implementers that may not generalize to other facilities. The project was an implemented in a SNF with a highly motivated administrator and medical director that were eager to collaborate on innovative projects. SNFs have many competing priorities, including staff turnover and the challenge of regulatory requirements, and may not have the ability to replicate a time-intensive project without support from administrative and physician leadership. Another limitation stems from the small population of implementers, leaving the evaluation to be based on only five interviews. A small number of implementers, however, meant that the entire population was surveyed rather than a potentially biased sample. Additionally, the development of the interview guide serves as a foundation for evaluation in future implementation projects. Larger studies implementing similar models in several SNFs will be needed to validate our observations. These should account for SNF baseline characteristics and clinician availability to analyze the impact on implementation efforts. The relatively smaller size of the SNF involved in this project meant that fewer resources were required for assuring patient and staff education. A larger scale adoption of our model will require resources for ongoing staff and patient education and for implementing team rounds.

The Affordable Care Act and the resulting models of care requiring accountability for patients across settings have motivated stakeholders, particularly hospital systems, to invest in SNFs that are willing to be active partners in providing high quality care. Hospital systems may need to share in some of the costs of implementing QI projects aimed at reducing readmissions. The SHU model is an example of a hospital-SNF collaboration to improve care for a common and serious medical condition -- a win/win strategy for both systems.

\section{Implications}

Despite known best practices, there are serious gaps in the provision of high quality care in many health care settings, including SNFs. The typical research-to-implementation timeline of 17 years (Brownson \& Jones, 2009) is unacceptable; implementation science aims to expedite the evolution of 
Running Head: IMPLEMENTATION OF HEART FAILURE CARE IN A NURSING FACILITY

healthcare systems by developing and testing new models of care (Newhouse, Bobay, Dykes, Stevens, \& Titler, 2013). In this context, our research offers a unique glimpse into the strategies for the implementation of a model focused on a common, morbid condition in the SNF environment. It may offer lessons for teams who are aiming to implement new models in the complex SNF environment and improve care for a very vulnerable patient population. 
Running Head: IMPLEMENTATION OF HEART FAILURE CARE IN A NURSING FACILITY

Table 1.Skilled Heart Unit Components

\begin{tabular}{|c|c|c|}
\hline & SHU target goal & Methods to achieve goal \\
\hline & $\begin{array}{l}\text { Implement best practices for hospital } \\
\text { to SNF and home transitional care }\end{array}$ & $\begin{array}{ll}- & \text { Communication systems } \\
- & \text { Nurse assessment } \\
- & \text { Advance care planning } \\
- & \text { Cardiologist appointments }\end{array}$ \\
\hline & $\begin{array}{l}\text { Utilize standardized assessments and } \\
\text { care protocols based on HF guidelines: }\end{array}$ & $\begin{array}{ll} & \text { Initial assessment (Ejection fraction, } \\
\text { vaccination status, diuretic and ACE- } \\
\text { inhibitor use, weights, and low } \\
\text { sodium diet) } \\
\text { - } & \text { Daily weights } \\
- & \text { Daily clinical rounds } \\
- & \text { Weekly HF clinical visit with } \\
& \text { progress notes }\end{array}$ \\
\hline & $\begin{array}{l}\text { Incorporate patient and family } \\
\text { education about HF symptoms and } \\
\text { treatment: }\end{array}$ & $\begin{array}{ll}- & \text { Session 1: Nurse driven HF } \\
\text { education with HFSA approved info } \\
\text { - } \quad \text { Session 2: Facility Nurse Practitioner } \\
\text { driven Individualized education about } \\
\text { weights, meds, warning symptoms at } \\
\text { the time of discharge }\end{array}$ \\
\hline & Conduct interdisciplinary meetings: & $\begin{array}{l}\text { Attendees - Clinician, Nurse Champion, } \\
\text { Therapy Staff, Nursing Director, Dietician, } \\
\text { Nurse Aides } \\
\text { Discussions: } \\
\quad \text { - Individual care and social support } \\
\quad \text { issues } \\
-\quad \text { Root cause analysis of transfers }\end{array}$ \\
\hline
\end{tabular}

HF: Heart Failure; HFSA: Heart Failure Society of America; SNF: Skilled Nursing Facility 
Figure 1. SHU Care Processes and Tools Utilized.

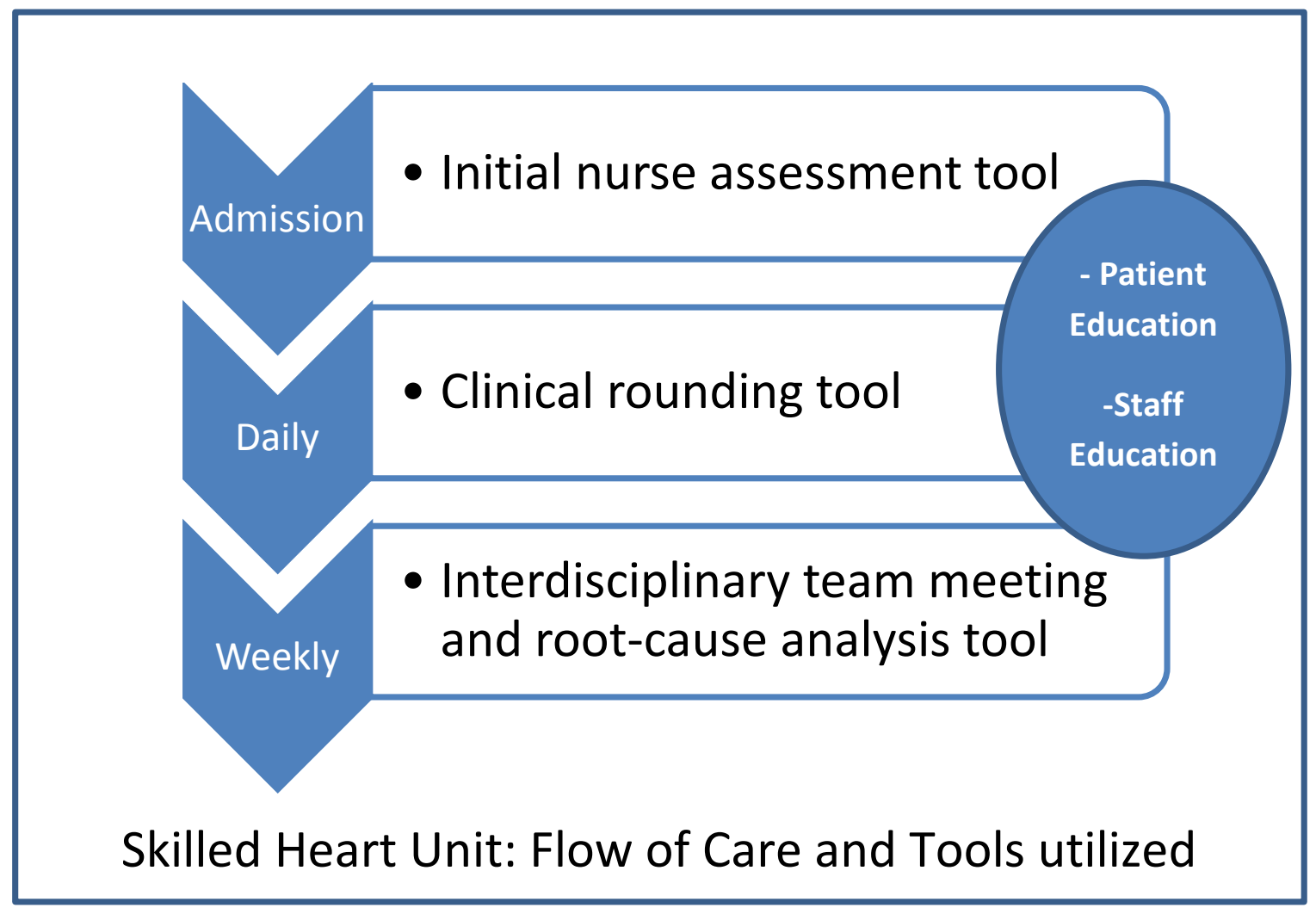




\section{Running Head: IMPLEMENTATION OF HEART FAILURE CARE IN A NURSING FACILITY}

\section{References}

Allen, L. A., Hernandez, A. F., Peterson, E. D., Curtis, L. H., Dai, D., Masoudi, F. A., . . Fonarow, G. C. (2011). Discharge to a skilled nursing facility and subsequent clinical outcomes among older patients hospitalized for heart failure. Circulation: Heart Failure, 4, 293-300. doi:

\subsection{1/CIRCHEARTFAILURE.110.959171}

Anderson, R. A., Issel, L. M., \& McDaniel Jr, R. R. (2003). Nursing homes as complex adaptive systems: relationship between management practice and resident outcomes. Nursing Research, 52, 12-21.

Arling, G., Cooke, V., Lewis, T., Perkins, A., Grabowski, D. C., \& Abrahamson, K. (2013). Minnesota's provider-initiated approach yields care quality gains at participating nursing homes. Health Affairs, 32, 1631-1638. doi: 10.1377/hlthaff.2013.0294

Boustani, M. A., Munger, S., Gulati, R., Vogel, M., Beck, R. A., \& Callahan, C. M. (2010). Selecting a change and evaluating its impact on the performance of a complex adaptive health care delivery system. Journal of Clinical Interventions in Aging, 5, 141-148.

Boustani, M. A., Sachs, G. A., Alder, C. A., Munger, S., Schubert, C. C., Guerriero Austrom, M., . . Callahan, C. M. (2011). Implementing innovative models of dementia care: The Healthy Aging Brain Center. Aging \& Mental Health, 15, 13-22. doi: 10.1080/13607863.2010.496445

Boxer, R. S., Dolansky, M. A., Frantz, M. A., Prosser, R., Hitch, J. A., \& Pina, I. L. (2012). The Bridge Project: improving heart failure care in skilled nursing facilities. Journal of the American Medical Directors Association, 13, 83 e81-87. doi: 10.1016/j.jamda.2011.01.005

Boyde, M., Turner, C., Thompson, D. R., \& Stewart, S. (2011). Educational interventions for patients with heart failure: a systematic review of randomized controlled trials. Journal of Cardiovascular Nursing, 26, E27-35. doi: 10.1097/JCN.0b013e3181ee5fb2

Callahan, C. M., Boustani, M. A., Weiner, M., Beck, R. A., Livin, L. R., Kellams, J. J., . . Hendrie, H. C. (2011). Implementing dementia care models in primary care settings: The Aging Brain Care Medical Home. Aging\& Mental Health, 15, 5-12. doi: 10.1080/13607861003801052 


\section{Running Head: IMPLEMENTATION OF HEART FAILURE CARE IN A NURSING FACILITY}

Counsell, S. R., Callahan, C. M., Clark, D. O., Tu, W., Buttar, A. B., Stump, T. E., \& Ricketts, G. D. (2007). Geriatric care management for low-income seniors: a randomized controlled trial. The Journal of the American Medical Association, 298, 2623-2633. doi: 10.1001/jama.298.22.2623

Counsell, S. R., Holder, C. M., Liebenauer, L. L., Palmer, R. M., Fortinsky, R. H., Kresevic, D. M., .. . Landefeld, C. S. (2000). Effects of a multicomponent intervention on functional outcomes and process of care in hospitalized older patients: a randomized controlled trial of Acute Care for Elders (ACE) in a community hospital. Journal of the American Geriatrics Society, 48, 15721581.

Daamen, M. A., Schols, J. M., Jaarsma, T., \& Hamers, J. P. (2010). Prevalence of heart failure in nursing homes: a systematic literature review. Scandinavian Journal of Caring Sciences, 24, 202-208. doi: 10.1111/j.1471-6712.2009.00708.x

Dolansky, M. A., Hitch, J. A., Pina, I. L., \& Boxer, R. S. (2013). Improving Heart Failure Disease Management in Skilled Nursing Facilities: Lessons Learned. Clinical Nursing Research, 22, 432447. doi: $10.1177 / 1054773813485088$

Foebel, A. D., Heckman, G. A., Ji, K., Dubin, J. A., Turpie, I. D., Hussack, P., \& McKelvie, R. S. (2013). Heart failure--related mortality and hospitalization in the year following admission to a long-term care facility: the geriatric outcomes and longitudinal decline in heart failure (GOLD-HF) study. Journal of Cardiac Failure, 19, 468-477. doi: 10.1016/j.cardfail.2013.05.003

Gheorghiade, M., Vaduganathan, M., Fonarow, G. C., \& Bonow, R. O. (2013). Rehospitalization for heart failure: problems and perspectives. Journal of the American College of Cardiology, 61, 391403. doi: 10.1016/j.jacc.2012.09.038

Harrington, C. C. (2008). Assessing heart failure in long-term care facilities. Journal of Gerontological Nursing, 34, 9-14.

Heckman, G. A., Misiaszek, B., Merali, F., Turpie, I. D., Patterson, C. J., Flett, N., \& McKelvie, R. S. (2004). Management of heart failure in Canadian long-term care facilities. Canadian Journal of Cardiology, 20, 963-969. 


\section{Running Head: IMPLEMENTATION OF HEART FAILURE CARE IN A NURSING FACILITY}

Horn, S. D., Sharkey, S. S., Hudak, S., Smout, R. J., Quinn, C. C., Yody, B., \& Fleshner, I. (2010). Beyond CMS quality measure adjustments: identifying key resident and nursing home facility factors associated with quality measures. Journal of the American Medical Directors Association, 11, 500-505. doi: 10.1016/j.jamda.2009.10.008

Lang, J., Thomas, J. L., Bliese, P. D., \& Adler, A. B. (2007). Job demands and job performance: the mediating effect of psychological and physical strain and the moderating effect of role clarity. Journal of Occupational Health Psychology, 12, 116-124. doi: 10.1037/1076-8998.12.2.116

Lindenfeld, J., Albert, N. M., Boehmer, J. P., Collins, S. P., Ezekowitz, J. A., Givertz, M. M., .. . Walsh, M. N. (2010). HFSA 2010 Comprehensive Heart Failure Practice Guideline. Journal of Cardiac Failure, 16, e1-194. doi: 10.1016/j.cardfail.2010.04.004

Lu, H., While, A. E., \& Barriball, K. L. (2005). Job satisfaction among nurses: a literature review. International Journal of Nursing Studies, 42, 211-227. doi: 10.1016/j.ijnurstu.2004.09.003

McAlister, F. A., Lawson, F. M., Teo, K. K., \& Armstrong, P. W. (2001). A systematic review of randomized trials of disease management programs in heart failure. American Journal of Medicine, 110, 378-384.

McAlister, F. A., Stewart, S., Ferrua, S., \& McMurray, J. J. (2004). Multidisciplinary strategies for the management of heart failure patients at high risk for admission: a systematic review of randomized trials. Journal of the American College of Cardiology, 44, 810-819. doi: 10.1016/j.jacc.2004.05.055

Naylor, M. D., Brooten, D. A., Campbell, R. L., Maislin, G., McCauley, K. M., \& Schwartz, J. S. (2004). Transitional care of older adults hospitalized with heart failure: a randomized, controlled trial. Journal of the American Geriatrics Society, 52, 675-684. doi: 10.1111/j.1532-5415.2004.52202.x

Nazir, A., Unroe, K., Tegeler, M., Khan, B., Azar, J., \& Boustani, M. (2013). Systematic review of interdisciplinary interventions in nursing homes. Journal of the American Medical Directors Association, 14, 471-478. doi: 10.1016/j.jamda.2013.02.005 


\section{Running Head: IMPLEMENTATION OF HEART FAILURE CARE IN A NURSING FACILITY}

Newhouse, R., Bobay, K., Dykes, P. C., Stevens, K. R., \& Titler, M. (2013). Methodology issues in implementation science. Medical Care, 51, S32-40. doi: 10.1097/MLR.0b013e31827feeca

Stroebel, C. K., McDaniel, R. R., Jr., Crabtree, B. F., Miller, W. L., Nutting, P. A., \& Stange, K. C. (2005). How complexity science can inform a reflective process for improvement in primary care practices. The Joint Commission Journal on Quality and Patient Safety, 31, 438-446.

Wagner, E. H., Bennett, S. M., Austin, B. T., Greene, S. M., Schaefer, J. K., \& Vonkorff, M. (2005). Finding common ground: patient-centeredness and evidence-based chronic illness care. Journal of Alternative and Complementary Medicine, 11, S7-15. doi: 10.1089/acm.2005.11.s-7 